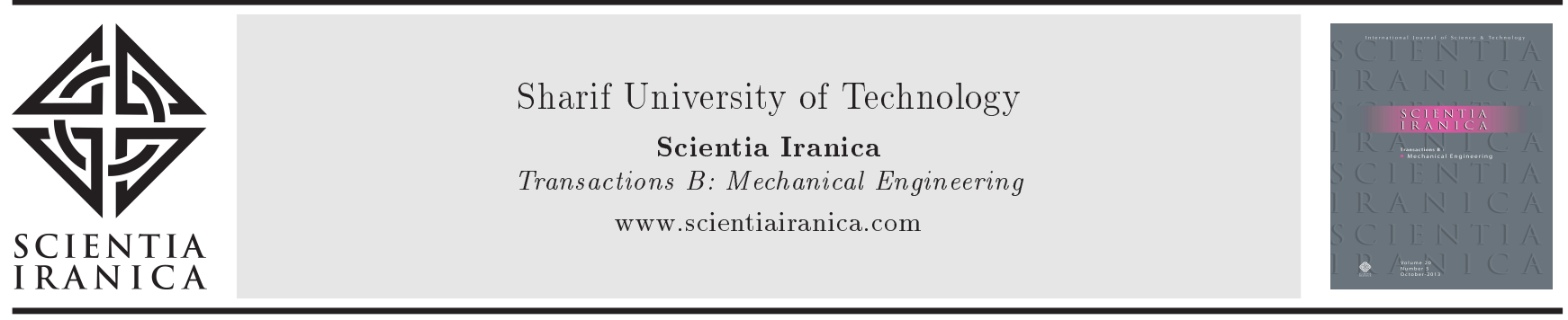

\title{
Group classification of the time-fractional Kaup-Kupershmidt equation
}

\author{
H. Jafari $i^{a, b, *}$ N. Kadkhoda ${ }^{c}$, M. Azadi ${ }^{a}$ and M. Yaghoubi ${ }^{\text {d }}$ \\ a. Department of Mathematics and Computer Science, University of Mazandaran, Babolsar, Iran. \\ b. Department of Mathematical Sciences, University of South Africa, UNISA 0003, South Africa. \\ c. Department of Mathematics, Faculty of Basic Sciences, Bozorgmehr University of Qaenat, Qaenat, Iran. \\ d. Department of Mathematics, Payame Noor University, Tehran, P.O. Box 19395-3697, Iran.
}

Received 26 October 2015; received in revised form 18 January 2016; accepted 27 February 2016

\author{
KEYWORDS \\ Fractional differential \\ equation; \\ Lie group; \\ Time-fractional \\ Kaup-Kupershmidt \\ equation; \\ Riemann-Liouville \\ derivative; \\ Group-invariant \\ solutions.
}

\begin{abstract}
Finding the symmetries of the nonlinear fractional differential equations plays an important role in study of fractional differential equations. In this manuscript, firstly, we are interested in finding the Lie point symmetries of the time-fractional KaupKupershmidt equation. Afterwards, by using the infinitesimal generators, we determine their corresponding invariant solutions.

(C) 2017 Sharif University of Technology. All rights reserved.
\end{abstract}

\section{Introduction}

The method of group analysis of differential equations was introduced by Sophus Lie about one hundred years ago [1-3]. Lie group theory is an efficient method that we use for analysis of Partial Differential Equations (PDEs). Lie symmetries method is an effective method to solve the problems of mathematical physics.

The Fractional Differential Equations (FDEs) have been studied by scientists since about thirty years ago. Many phenomena in nature can be described using the FDEs. The fractional differential equations arise in many fields of sciences such as, electrochemistry, physics, biology, mechanics, signal processing, and viscoelastic materials [4-12].

\footnotetext{
*. Corresponding author.

E-mail addresses: jafari@umz.ac.ir (H. Jafari);

kadkhoda@buqaen.ac.ir (N. Kadkhoda);

yaghoobim@yahoo.com (M. Yaghoubi)
}

Many articles have been presented to define fractional derivatives. The most important ones are the Caputo and the Riemann-Liouville derivatives. Each fractional derivative has some advantages and disadvantages. The Caputo derivative of a constant is zero, but Riemann-Liouville derivative of a constant is not. Many articles have been exhibited for finding the exact solutions of FDEs. There are many techniques and methods in these papers, which constitute the numerical and analytical solutions of FDEs. These methods include the fractional complex transform [13], the separating variables method [14], the variational iteration method [15], the first integral method [16], the homotopy analysis method [17], the homotopy perturbation Pade technique [18], the generalized differential transform method [19], the Hermit transform [20], etc. Many researchers obtained the exact solutions of many nonlinear PDEs using Lie group theory. But, the question may be asked here: Can we use this method for FDEs? Up to now, for FDEs, only a few works can be found in literature [21-27]. One of 
the difficulties of this type of problems originates in the non-local type of the fractional operators. Using the abovementioned research in our manuscript, we study the time-fractional Kaup-Kupershmidt equation, namely:

$$
\begin{aligned}
& D_{t}^{\alpha} u-u_{5 x}-10 u u_{3 x}-25 u_{x} u_{2 x}-20 u_{x} u^{2}=0, \\
& \quad t>0, \quad x \in R
\end{aligned}
$$

where $0<\alpha<1 ; u$ is a function of $(x, t)$ and $u \in C^{\infty}\left(R^{2}\right)$. The Kaup-Kupershmidt equation plays an important role in the nonlinear dispersive wave. Solitary waves propagate in nonlinear dispersive media. These waves preserve a stable form due to dynamic balance between the dispersive and nonlinear influences. The exact solutions of this equation have been presented in many articles, such as $[28,29]$.

The rest of our work is organized as follows. In Section 2, we present the analysis of the Lie Symmetry group of FDEs. Afterwards, in Section 3, we obtain the Lie point symmetries of the time-fractional Kaup-Kupershmidt equation. Finally, we obtain invariant solutions and reduced equations of this equation in Section 4. Discussion and conclusions are presented in Section 5.

\section{Description of the symmetry group analysis of FDEs}

Finding the exact solutions of the fractional differential equations is an important and difficult task. Therefore, much effort has been made to obtain the exact solutions of them. We recall that the symmetry is one of the most important concepts to study of the differential equations. Finding the exact solutions of differential equations using the fundamental method of the Lie symmetries has been used by many researchers. Invariance of the equations under transformation groups is the basic concept of the Lie theory. As it is known, there is the possibility of simplifying the differential equations if there are symmetries of the differential equations. We recall the works on this topic of Ovsiannikov [1], Olver [2], Ibragimov [3], Baumann [30], Bluman \& Anco [31], and You \& Zhang [32]. Now, we express the fractional Lie group method for finding infinitesimal functions of FPDEs. Let us assume an FPDE of the form:

$$
D_{t}^{\alpha} u=F\left(x, t, u_{(1)}, \cdots\right), \quad \alpha>0,
$$

where $u$ is a function of independent variables; $x, t$, and $D_{t}^{\alpha}$ can be defined as follows.

Definition $1[4,6] . \quad D_{t}^{\alpha}$ is the Riemann-Liouville fractional derivative operator defined by:

$$
D_{t}^{\alpha} u=\left\{\begin{array}{c}
\frac{\partial^{m} u}{\partial t^{m}}, \\
\alpha=m \in N, \\
\frac{1}{\Gamma(m-\alpha)} \frac{\partial^{m}}{\partial t^{m}} \int_{0}^{t} \frac{u(\tau, x)}{(t-\tau)^{\alpha+1-m}} d \tau ; \\
m-1 \leq \alpha<m, m \in N .
\end{array}\right.
$$

Similar to the discussion on PDEs [2,33], we can write:

$$
D_{\bar{t}}^{\alpha} \bar{u}=D_{t}^{\alpha} u+\varepsilon\left[\eta_{t}^{(\alpha)}\left(x, t, u, u_{(\alpha)}, u_{(1)}, \cdots\right)\right]+O\left(\varepsilon^{2}\right),
$$

here, $\eta_{t}^{(\alpha)}$ is given by the prolongation formula [22]:

$$
\begin{aligned}
\eta_{t}^{(\alpha)}= & D_{t}^{\alpha}(\eta)+\xi^{x} D_{t}^{\alpha}\left(u_{x}\right)-D_{t}^{\alpha}\left(\xi^{x} u_{x}\right) \\
& +D_{t}^{\alpha}\left(D_{t}\left(\xi^{t}\right) u\right)-D_{t}^{\alpha+1}\left(\xi^{t} u\right)+\xi^{t} D_{t}^{\alpha+1} u,(5)
\end{aligned}
$$

where $D_{t}$ is the total derivative operator defined as:

$$
\begin{aligned}
D_{t}= & \frac{\partial}{\partial t}+u_{t} \frac{\partial}{\partial u}+u_{x t} \frac{\partial}{\partial u_{x}}+u_{t t} \frac{\partial}{\partial u_{t}} \\
& +u_{x x t} \frac{\partial}{\partial u_{x x}}+\cdots
\end{aligned}
$$

Simplifying Eq. (5) using the Leibnitz formula [34]:

$$
D_{t}^{\alpha}[f(t) g(t)]=\sum_{n=0}^{\infty}\left(\begin{array}{l}
\alpha \\
n
\end{array}\right) D_{t}^{\alpha-n} f(t) D_{t}^{n} g(t),
$$

$$
\alpha>0
$$

where:

$$
\begin{aligned}
\left(\begin{array}{l}
\alpha \\
n
\end{array}\right) & =\frac{(-1)^{n-1} \alpha \Gamma(n-\alpha)}{\Gamma(1-\alpha) \Gamma(n+1)}, \\
\Gamma(z) & =\int_{0}^{\infty} t^{z-1} e^{-t} d t
\end{aligned}
$$

we can write [35]:

$$
\begin{aligned}
\eta_{t}^{(\alpha)} & =\frac{\partial^{\alpha} \eta}{\partial t^{\alpha}}+\left(\eta_{u}-\alpha D_{t}\left(\xi^{t}\right)\right) \frac{\partial^{\alpha} u}{\partial t^{\alpha}}-u \frac{\partial^{\alpha} \eta_{u}}{\partial t^{\alpha}} \\
& +\sum_{n=1}^{\infty}\left[\left(\begin{array}{l}
\alpha \\
n
\end{array}\right) \frac{\partial^{n}\left(\eta_{u}\right)}{\partial t^{n}}-\left(\begin{array}{c}
\alpha \\
n+1
\end{array}\right) D_{t}^{n+1}\left(\xi^{t}\right)\right] D_{t}^{\alpha-n}(u) \\
& -\sum_{n=1}^{\infty}\left(\begin{array}{l}
\alpha \\
n
\end{array}\right) D_{t}^{\alpha-n}\left(u_{x}\right) D_{t}^{n}\left(\xi^{x}\right)
\end{aligned}
$$

We have a definition as follows.

Definition 2. The equations for finding coefficients of the infinitesimal operator $X$ are given below: 
$X^{(\alpha)}\left[D_{t}^{\alpha} u-F\left(x, t, u, u_{(1)}, \cdots\right)\right]_{D_{t}^{\alpha} u=F\left(x, t, u_{(1)}, \cdots\right)}=0$

where:

$$
\begin{aligned}
X^{(\alpha)}= & \xi^{x}(x, t, u) \frac{\partial}{\partial x}+\xi^{t}(x, t, u) \frac{\partial}{\partial t}+\eta(x, t, u) \frac{\partial}{\partial u} \\
& +\eta_{i}^{(1)}\left(x, t, u, u_{(1)}\right) \frac{\partial}{\partial u_{i}}+\cdots \\
& +\eta_{i_{1}, i_{2} \cdots, i_{k}}^{(k)}\left(x, t, u, u_{(1)}, \cdots, u_{(k)}\right) \frac{\partial}{\partial u_{i_{1}, i_{2} \cdots, i_{k}}} \\
& +\eta_{t}^{(\alpha)}\left(x, t, u, \cdots, u_{(\alpha)}, \cdots\right) \frac{\partial}{\partial u_{t}^{(\alpha)}} .
\end{aligned}
$$

Expanding Eq. (10) using Eq. (11) and preceding relations, we obtain the determining equations. As a result, these obtained equations yield Lie symmetries.

\section{Application of fractional Lie symmetries to the time-fractional Kaup-Kupershmidt equation}

Here, we employ this method for the time-fractional Kaup-Kupershmidt equation:

$$
\begin{aligned}
& D_{t}^{\alpha} u-u_{5 x}-10 u u_{3 x}-25 u_{x} u_{2 x}-20 u_{x} u^{2}=0, \\
& t>0, \quad 0<\alpha<1 .
\end{aligned}
$$

We search the infinitesimal generator of Eq. (12).

Theorem 1. Lie symmetries of the time fractional Kaup-Kupershmidt equation (Eq. (12)) are:

1. If $\alpha \neq \frac{1}{2}, \frac{4}{5}$, then we have:

$$
\xi^{x}=c_{1} \alpha x+c_{2}, \quad \xi^{t}=5 c_{1} t, \quad \eta_{u}=-2 c_{1} \alpha u,
$$

where $c_{1}$ and $c_{2}$ are two arbitrary constants. Therefore, the infinitesimal generators are given by:

$$
X_{1,1}=\frac{\partial}{\partial x}, \quad X_{1,2}=\alpha x \frac{\partial}{\partial x}+5 t \frac{\partial}{\partial t}-2 \alpha u \frac{\partial}{\partial u} .
$$

2. If $\alpha=\frac{1}{2}$, then we have:

$$
\xi^{x}=c_{1} x+c_{2}, \quad \xi^{t}=10 c_{1} t, \quad \eta_{u}=-2 c_{1} u,
$$

where $c_{1}$ and $c_{2}$ are two arbitrary constants. Therefore, the infinitesimal generators are given by:

$$
X_{2,1}=\frac{\partial}{\partial x}, \quad X_{2,2}=x \frac{\partial}{\partial x}+10 t \frac{\partial}{\partial t}-2 u \frac{\partial}{\partial u} .
$$

3. If $\alpha=\frac{4}{5}$, then we have:

$$
\xi^{x}=4 c_{1} x+c_{2}, \quad \xi^{t}=25 c_{1} t, \quad \eta_{u}=-8 c_{1} u,
$$

where $c_{1}$ and $c_{2}$ are three arbitrary constants. Therefore, the infinitesimal generators are given by:

$$
X_{3,1}=\frac{\partial}{\partial x}, \quad X_{3,2}=4 x \frac{\partial}{\partial x}+25 t \frac{\partial}{\partial t}-8 u \frac{\partial}{\partial u} .
$$

Proof. Let us assume the one-parameter Lie group of infinitesimal transformations in $x, t, u$ given by:

$$
\begin{aligned}
& x^{*}=x+\varepsilon \xi^{x}(x, t, u)+O\left(\varepsilon^{2}\right), \\
& t^{*}=t+\varepsilon \xi^{t}(x, t, u)+O\left(\varepsilon^{2}\right), \\
& u^{*}=u+\varepsilon \eta_{u}(x, t, u)+O\left(\varepsilon^{2}\right),
\end{aligned}
$$

where $\varepsilon$ is the group parameter, and the Lie algebra of Kaup-Kupershmidt equation is spanned by vector fields:

$$
X=\xi^{x}(x, t, u) \frac{\partial}{\partial x}+\xi^{t}(x, t, u) \frac{\partial}{\partial t}+\eta_{u}(x, t, u) \frac{\partial}{\partial u},
$$

where:

$$
\xi^{x}=\left.\frac{d x^{*}}{d \varepsilon}\right|_{\varepsilon=0}, \quad \xi^{t}=\left.\frac{d t^{*}}{d \varepsilon}\right|_{\varepsilon=0}, \quad \eta_{u}=\left.\frac{d u^{*}}{d \varepsilon}\right|_{\varepsilon=0} .
$$

Applying $X^{(\alpha)}$ to Eq. (12), we have:

$$
\begin{aligned}
X^{(\alpha)} & {\left[D_{t}^{\alpha} u-u_{5 x}-10 u u_{3 x}-25 u_{x} u_{2 x}\right.} \\
& \left.-20 u_{x} u^{2}\right]_{D_{t}^{\alpha} u-u_{5 x}-10 u u_{3 x}-25 u_{x} u_{2 x}-20 u_{x} u^{2}=0} \\
& =0
\end{aligned}
$$

where $X^{(\alpha)}$ is given by Eq. (11). Expanding Eq. (15), and solving the obtained system using the Maple, we obtain the Lie point symmetries for the time-fractional Kaup-Kupershmidt equation. If $\alpha \neq \frac{1}{2}, \frac{4}{5}$, then we have:

$$
\xi^{x}=c_{1} \alpha x+c_{2}, \quad \xi^{t}=5 c_{1} t, \quad \eta_{u}=-2 c_{1} \alpha u .
$$

Therefore, the infinitesimal generators are given by:

$$
X_{1,1}=\frac{\partial}{\partial x}, \quad X_{1,2}=\alpha x \frac{\partial}{\partial x}+5 t \frac{\partial}{\partial t}-2 \alpha u \frac{\partial}{\partial u} .
$$

We now apply this argument again, with $\alpha=\frac{1}{2}$, to obtain:

$$
\xi^{x}=c_{1} x+c_{2}, \quad \xi^{t}=10 c_{1} t, \quad \eta_{u}=-2 c_{1} u .
$$

Therefore, the infinitesimal generators are given by:

$$
X_{2,1}=\frac{\partial}{\partial x}, \quad X_{2,2}=x \frac{\partial}{\partial x}+10 t \frac{\partial}{\partial t}-2 u \frac{\partial}{\partial u} .
$$

In the same manner, for $\alpha=\frac{4}{5}$, we can obtain:

$$
\xi^{x}=4 c_{1} x+c_{2}, \quad \xi^{t}=25 c_{1} t, \quad \eta_{u}=-8 c_{1} u .
$$

Therefore, the infinitesimal generators are given by:

$$
X_{3,1}=\frac{\partial}{\partial x}, \quad X_{3,2}=4 x \frac{\partial}{\partial x}+25 t \frac{\partial}{\partial t}-8 u \frac{\partial}{\partial u} .
$$

The proof is completed. 


\section{Invariant solutions and the reduced equations of the time-fractional Kaup-Kupershmidt equation}

The time-fractional Kaup-Kupershmidt equation is expressed by the coordinates $(x, t, u)$; thus, we want to reduce it using new coordinates. By introducing invariants $(r, z)$, we obtain the new coordinates corresponding to the infinitesimal symmetry generator and we can reduce the mentioned equation [36]. Consider a Lie point symmetry:

$$
X=\xi^{x}(x, t, u) \frac{\partial}{\partial x}+\xi^{t}(x, t, u) \frac{\partial}{\partial t}+\eta_{u}(x, t, u) \frac{\partial}{\partial u},
$$

of the time-fractional Kaup-Kupershmidt equation:

$$
\begin{aligned}
& D_{t}^{\alpha} u-u_{5 x}-10 u u_{3 x}-25 u_{x} u_{2 x}-20 u_{x} u^{2}=0 \\
& \quad t>0, \quad 0<\alpha<1
\end{aligned}
$$

Under the one-parameter group generated by $X$, the invariant solutions are obtained as follows. Two linearly independent invariants $r=\varphi(x, t)$ and $z=\psi(x, t)$ can be calculated by solving the first-order quasi-linear PDE:

$$
\begin{aligned}
X(J)= & \xi^{x}(x, t, u) \frac{\partial(J)}{\partial x}+\xi^{t}(x, t, u) \frac{\partial(J)}{\partial t} \\
& +\eta_{u}(x, t, u) \frac{\partial(J)}{\partial u}=0,
\end{aligned}
$$

or its characteristic equations:

$$
\frac{d x}{\xi^{x}(x, t, u)}=\frac{d t}{\xi^{t}(x, t, u)}=\frac{d u}{\eta_{u}(x, t, u)} .
$$

Then, we write one of the invariants as a function of the other, for example:

$$
z=f(r)
$$

and solve Eq. (16) for $u$. Finally, the expression of $u$ is substituted in Eq. (12) and a fractional ODE is obtained for the unknown function $f$. With this procedure, we can reduce the number of independent variables by one. Now, we obtain the corresponding invariants and present the reduced nonlinear fractional ordinary differential equations. Finally, we obtain the corresponding group invariant solutions of the fractional Kaup-Kupershmidt equation as follows:

Case 1: $\quad 0<\alpha<1, \alpha \neq \frac{1}{2}, \frac{4}{5}, X_{1,1}=\partial_{x}$.

In this case, the corresponding invariants are given by:

$$
r=t, \quad z=u \text {. }
$$

A solution to our equation becomes:

$$
z=f(r) \Rightarrow u=f(t)
$$

We substitute Eq. (18) into Eq. (12) in order to determine $f(r)$. Then, $f(r)$ fulfils the following differential equation:

$$
\frac{d^{\alpha} f(t)}{d t^{\alpha}}=0
$$

The solution of the Eq. (19), by using Laplace transform, is given by [6]:

$$
f(t)=\frac{k}{\Gamma(\alpha)} t^{\alpha-1}
$$

where $k$ is a constant and $\Gamma(\alpha)$ is given by Eq. (8).

Case 2: $\quad 0<\alpha<1, \alpha \neq \frac{1}{2}, \frac{4}{5}, X_{1,2}=\alpha x \frac{\partial}{\partial x}+5 t \frac{\partial}{\partial t}-$ $2 \alpha u \frac{\partial}{\partial u}$.

For this case, the corresponding invariants are given below:

$$
r=t x^{-\frac{5}{\alpha}}, \quad z=x^{2} u
$$

Then, a solution to our equation has the form:

$$
z=f(r) \Rightarrow u=x^{-2} f\left(t x^{-\frac{5}{\alpha}}\right)
$$

and we substitute it into Eq. (12) to determine $f(r)$. Thus, $f(r)$ is satisfied in the following equation:

$$
\begin{aligned}
& \alpha^{5} \frac{\partial^{\alpha} f}{\partial r^{\alpha}}+k_{1} f(r)+k_{2} f(r)^{2}+k_{3} f(r)^{3}+k_{4} r f^{\prime}(r) \\
& \quad+k_{5} r f(r) f^{\prime}(r)+k_{6} r f(r)^{2} f^{\prime}(r)+k_{7} r^{2} f^{\prime}(r)^{2} \\
& \quad+k_{8} r^{2} f^{\prime \prime}(r)+k_{9} r^{2} f(r) f^{\prime \prime}(r)+k_{10} r^{3} f^{\prime}(r) f^{\prime \prime}(r) \\
& \quad+k_{11} r^{3} f^{(3)}(r)+k_{12} r^{3} f(r) f^{(3)}(r)+k_{13} r^{4} f^{(4)}(r) \\
& \quad+k_{14} r^{5} f^{(5)}(r)=0,
\end{aligned}
$$

where $k_{i}=h_{i}(\alpha)(i=1,2, \ldots, 14)$ are constants.

Case 3: $\quad \alpha=\frac{1}{2}, X_{22}=x \frac{\partial}{\partial x}+10 t \frac{\partial}{\partial t}-2 u \frac{\partial}{\partial u}$.

The corresponding invariants for $\alpha=\frac{1}{2}$ and $X_{2,2}$ can be written as:

$$
r=t x^{-10}, \quad z=x^{2} u \text {. }
$$

As a result, we obtain:

$$
z=f(r) \Rightarrow u=x^{-2} f\left(t x^{-10}\right)
$$


by substituting Eq. (24) into Eq. (12), we are able to determine $f(r)$ as solution to the following differential equation:

$$
\begin{aligned}
\frac{1}{20} \frac{\partial^{\alpha} f}{\partial r^{\alpha}} & +5000 r^{5} f^{(5)}(r)+60000 r^{4} f^{(4)}(r) \\
& +1250 r^{3} f^{\prime}(r) f^{\prime \prime}(r)+500 r^{3} f(r) f^{(3)}(r) \\
& +192750 r^{3} f^{(3)}(r)+1875 r^{2} f^{\prime}(r)^{2} \\
& +2200 r^{2} f(r) f^{\prime \prime}(r)+171150 r^{2} f^{\prime \prime}(r) \\
& +10 r f(r)^{2} f^{\prime}(r)+1530 r f(r) f^{\prime}(r) \\
& +26172 r f^{\prime}(r)+2 f(r)^{3}+27 f(r)^{2}+36 f(r) \\
= & 0 .
\end{aligned}
$$

Case 4: $\quad \alpha=\frac{4}{5}, X_{3,2}=4 x \frac{\partial}{\partial x}+25 t \frac{\partial}{\partial t}-8 u \frac{\partial}{\partial u}$. forms:

The invariants in this case have the following

$$
r=t x^{-\frac{25}{4}}, \quad z=x^{2} u .
$$

As a result, we obtain:

$$
z=f(r) \Rightarrow u=x^{-2} f\left(t x^{-\frac{25}{4}}\right) \text {. }
$$

By substituting Eq. (26) into Eq. (12), we conclude that $f(r)$ has to satisfy the following differential equation:

$$
\begin{aligned}
\frac{1024}{5} & \frac{\partial^{\alpha} f}{\partial r^{\alpha}}+1953125 r^{5} f^{(5)}(r)+25781250 r^{4} f^{(4)}(r) \\
& +1250000 r^{3} f^{\prime}(r) f^{\prime \prime}(r)+500000 r^{3} f(r) f^{(3)}(r) \\
& +94078125 r^{3} f^{(3)}(r)+2250000 r^{2} f^{\prime}(r)^{2} \\
& +2620000 r^{2} f(r) f^{\prime \prime}(r)+100936875 r^{2} f^{\prime \prime}(r) \\
& +25600 r f(r)^{2} f^{\prime}(r)+2464800 r f(r) f^{\prime}(r) \\
& +21929445 r f^{\prime}(r)+8192 f(r)^{3}+110592 f(r)^{2} \\
& +147456 f(r) \\
= & 0 .
\end{aligned}
$$

\section{Conclusion}

In the present study, we investigated the efficiency of the classical Lie symmetry group analysis for the fractional differential equations. The fractional Lie symmetries method was use for application to the timefractional Kaup-Kupershmidt equation with RiemannLiouville derivative, and we found the Lie point symmetries group of this equation. As an application of the infinitesimal symmetries, we have shown that time-fractional Kaup-Kupershmidt equation can be obtained as a nonlinear ODE of fractional order. Finally, some group invariant solutions have been obtained in explicit form as well.

\section{References}

1. Ovsyannikov, L.V., Group Analysis of Differential Equations, Academic Pres, New York (1982).

2. Olver, P.J. "Applications of Lie groups to differential equations", Graduate Texts in Mathematics, 107, 2nd Edn., Springer-Verlag, Berlin (1993).

3. Ibragimov, N.H., Handbook of Lie Group Analysis of Differential Equations, 1, 2, 3, CRC Press, Boca Raton, Ann Arbor, London, Tokyo (1994, 1995, 1996).

4. Baleanu, D., Diethelm, K., Scalas, E. and Trujillo, J.J. "Fractional calculus models and numerical methods", Series on Complexity, Nonlinearity and Chaos World Scientific (2012).

5. Kilbas, A.A., Srivastava, H.M. and Trujillo, J.J. "Theory and applications of fractional differential equations", North-Holland Mathematics Studies, 204, Elsevier Science B.V., Amsterdam, The Netherlands, (2006).

6. Podlubny, I. "Fractional differential equations: an introduction to fractional derivatives, fractional differential equations, to Methods of Their Solution and some of Their Applications", Mathematics in Science and Engineering, 198, Academic Press, San Diego, USA (1999).

7. Machado, J.A.T. and Galhano, A. "Approximating fractional derivatives in the perspective of system control", Nonlinear Dyn., 56, pp. 401-407 (2009).

8. Mainardi, F. "The fundamental solutions for the fractional diffusion-wave equation", Appl. Math. Lett., 9, pp. 23-28 (1996).

9. Oldham, K.B. and Spanier, J., The Fractional Calculus, Academic Press, New York (1974).

10. Samko, G,. Kilbas, A.A. and Marichev, O.I., Fractional Integrals and Derivatives: Theory and Applications, Gordon and Breach, Yverdon (1993).

11. Wu, G.C. and Baleanu, D. "Discrete fractional logistic map and its chaos", Nonlinear Dyn., 75(1-2), pp. 283287 (2014).

12. Wu, F. and Liu, J.F. "Discrete fractional creep model of salt rock", J. Comput. Complex. Appl., 2(1), pp. 1-6 (2016).

13. He, J.H. "Analytical methods for thermal science Anelementary introduction", Thermal Science, 15(5), pp. 1-3 (2011).

14. Chen, J., Liu, F. and Anh, V. "Analytical solution for the time-fractional telegraph equation by the method of separating variables", J. Math. Anal. Appl., 338(2), pp. 1364-1377 (2008). 
15. He, J.H., Wu, G.C. and Austin, F. "The variational iteration method which should be followed", Nonlinear Sci. Lett. A, 1(1), pp. 1-30 (2010).

16. Lu, B. "The first integral method for some time fractional differential equations", J. Math. Anal. Appl., 395, pp. 684-693 (2012).

17. Dehghan, M., Manafian, J. and Saadatmandi, A. "Solving nonlinear fractional partial differential equations using the homotopy analysis method", Numer. Meth. Part. D. E., 26(2), pp. 448-479 (2010).

18. Jafari, H., Kadkhoda, N., Tajadodi, H. and Hosseini Matikolai, S.A. "Homotopy perturbation Pade technique for solving fractional Riccati differential equations", Int. J. Nonlin. Sci.Numer. Simul., 11, pp. 271-275 (2010).

19. Garg, M. and Manohar, P. "Analytical solution of the reaction-diffusion equation with space-time fractional derivatives by means of the generalized differential transform method", Kuwait Journal of Science, 40(1), pp. 23-34 (2013).

20. Ghany, H. and Hyder, A. "Exact solutions for the wick-type stochastic time-fractional KdV equations", Kuwait Journal of Science, 41(1), pp. 75-84 (2014).

21. Buckwar, E. and Luchko, Yu. "Invariance of a partial differential equation of fractional order under the Lie group of scaling transformations", J. Math. Anal. Appl., 227, pp. 81-97 (1998).

22. Gazizov, R.K., Kasatkin, A.A. and Lukashchuk, S.Y. "Symmetry properties of fractional diffusion equations", Phys. Scr. T136, 014016 (2009).

23. Jafari, H., Kadkhoda, N. and Baleanu, D. "Fractional Lie group method of the time-fractional Boussinesq equation", Nonlinear Dynamics, 81(3), pp. 1569-1574 (2015).

24. Kasatkin, A.A. "Symmetry properties for systems of two ordinary fractional differential equations", Ufa Mathematical Journal, 4, pp. 65-75 (2012).

25. Liu, H.Z. "Complete group classifications and symmetry reductions of the fractional fifth-order KdV types of equations", Stud. Appl. Math., 131, pp. 317-330 (2013).

26. Wei, F. and Lina, Ji. "Symmetry analysis and groupinvariant solutions to inhomogeneous nonlinear diffusion equation", Communications in Nonlinear Science and Numerical Simulation, 28(1-3), pp. 50-65 (2015).

27. Wu, G.C. "A Fractional Lie group method for anomalous diffusion equations", Commun. Frac. Calc., 1, pp. 27-31 (2010).

28. Guzali. A., Manafian, J. and Jalali, J. "Application of homotopy analysis method for solving nonlinear fractional partial differential equations", Asian Journal of Fuzzy and Applied Mathematics, 2(2), pp. 89-102 (2014).

29. Usman, M. and Tauseef Mohyud-Din, S. "U-expansion method for 5th order Kaup Kuperschmidt and Lax equation of fractional order", International Journal of Modern Math. Sci., 9(2), pp. 63-81 (2014).
30. Baumann, G., Symmetry Analysis of Differential Equations with Mathematica, Telos, Springer Verlag, New York (2000).

31. Bluman, G.W. and Anco, S.C., Symmetry and Integration Methods for Differential Equations, SpringerVerlag, New York (2002).

32. You, F.C. and Zhang, J. "Lie superalgebras and multi-component superintegrable systems", J. Comput. Complex. Appl., 2(2), pp. 49-63 (2016).

33. Bluman, G.W. and Kumei, S. "Symmetries and differential equations", Appl. Math. Sci., 81, SpringerVerlag, New York (1989).

34. Wang, G.W., Liu, X.Q. and Zhang, Y.Y. "Lie symmetry analysis to the time fractional generalized fithorder KdV equation", Commun. Nonlin. Sci. Numer Simulat., 18(9), pp. 2321-2326 (2012).

35. Jefferson, G.F. and Carminati, J. "Frac sym: Automated symbolic computation of Lie symmetries of fractional differential equations", Comp. Phys. Commun., 185, pp. 430-441 (2014).

36. Nadjafikhah, M. and Ahangari, F. "Symmetry reduction of two-dimensional damped KuramotoSivashinsky equation", Commun. Theor. Phys., 56, pp. 211-217 (2011).

\section{Biographies}

Hossein Jafari is a Professor of Applied Mathematics in University of Mazandaran, Bababolsar, Iran, in 2005. In 2015, he was selected as one of the most cited Iranian researchers in Applied Mathematics by Thomson Reuters. His Research interests include fractional differential equations and dynamic systems.

Nematollah Kadkhoda received his $\mathrm{MSc}$ and $\mathrm{PhD}$ degrees in Applied Mathematics from University of Mazandaran, Babolsar, Iran. In 2015, he joined Bozorgmehr University of Qaenat, Qaenat, Iran, as Assistant Professor. His fields of research are mainly Lie symmetry and partial differential equations.

Marzieh Azadi received her MSc degree in Applied Mathematics from Hakim Sabzevari University, Sabzevar, Iran, in 2011. She is a PhD candidate under supervision of Prof. H. Jafari at University of Mazandaran. Her research field includes partial differential equations of fractional order and Lie symmetry method.

Maryam Yaghoubi is an Assistant Professor of Mathematics at Payame Noor University of Iran. Her research interest is analysis. She has published several research articles in different international journals. She has recently published a textbook on differential equation for university students. 